Historic, Archive Document

Do not assume content reflects current scientific knowledge, policies, or practices. 



\section{Wholesale Field Seed Price List}

(To the Trade)

L. L. Olds

720-724 Williamson St.

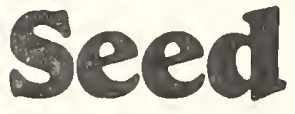

Today's Prices, F. O. B. Madison, subject to market change.

Bagss extra at value except where noted. Samples on application.

Terms, Net Cash. Cartage Free. Bags Not Returnable.
Company

Phone: Badger 1322

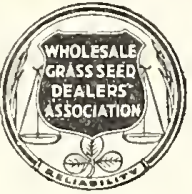

Madison Wisconsin July 29, 1927

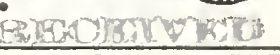

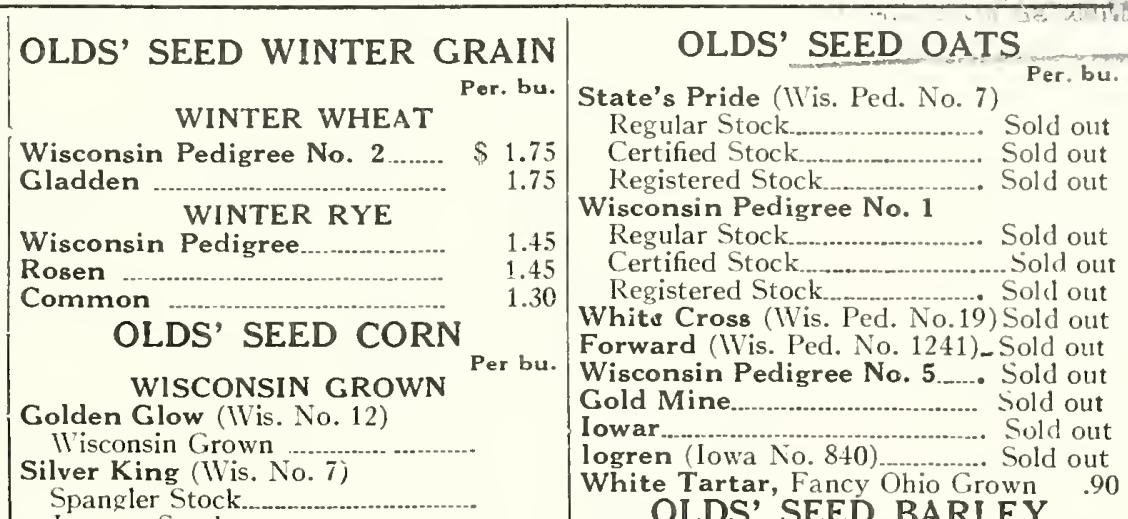

\section{CLOVERS}

Medium Clover (Wis, Minn, and ldaho) Lakes Brand.......................... Sold out OLD GOLD BRAND 43.50

Mammoth Clover (IVisconsin Grown) Lakes Brand OLD GOLD BRAND_.........45.00

Alsike Clover (Wisconsin Grown)

Lakes Brand (About $8 \%$ White) 28.00 OLD GOLD BRAND

White Dutch Clover (Wisconsin Grown) Lakes Brand (About $6 \%$ Alsike). 30.00 OLD GOLD BRAND 35.00

White Sweet Clover (Scarified) Lakes Brand.

OLD GOLD BRAND

Grundy County Sweet (Scarified)

OLD GOLD BRAND

Yellow Sweet (Scarified)

12.25

Hubam Sweet (Scarified)......... Sold ont

\section{ALFALFA}

Hardy Dakota No. 12 (60s and 1.50s) (Black Hills) (Sealed bags).......... 23.00

Hardy Montana, (60s)

(State Sealed and Certified).

Fancy ldaho.

Fancy Utah.

Fancy Kansas... OLD GOLD BRAND, Full bags 26.50 30s, 60 and 150s, Sealed

Blackfoot Idaho Grimm

Grade No. 1, Red Tag...

Grade Extra No. 1, Blue Tag . Sold out

(Blackfoot Grimm is State Certified, all in
Sealed 30 s and 60s, Sacks Free)

\section{TIMOTHY AND RED TOP}

\section{Timothy}

Lakes Brand

OLD GOLD BRAND

Timothy and Alsike (1-5 Alsike)

Red Top, Fancy Solid Seed.

\section{LIGHT GRASSES}

(Prices include sacks for all Light Grasses)

Kentucky Blue Grass

19 Pound Seed.

21 Pound Seed.

Orchard Grass.

Rye Grass

Perennial or English

Italian

Pacey's

American Grown

Bromus Inermis

Creeping Bent.

Creeping Fescue

Chewing's Fescue

Olds' Pasture Mixture

Olds' Meadow Mixture.

LAWN GRASS MIXTURES (Prices include sacks)

Old Gold, in bulk

Madison Parks, in bulk
Capital, in bulk

Shady Place, in bulk

ld Gold, in lb. cartons, case of 48

Madison Parks, in lb. cartons,

case of 48 .
Spangler Stock.

Other IIisconsin, $92 \%$ test

Other Wisconsin, $80 \%$

Murdock (Wis. No. 13)

Brueckner Stock.

Other Wisconsin.

Minnesota Grown

Cold Resistant Golden Glow

Jacques Stock

Other Northern Wisconsin, $74 \%$

Wisconsin No. 25

Clark's Yellow Dent (Wis. No. 1)

Improved Smut Nose Flint

Golden Glow NEBRASKA GROWN

First Year Wis. Seed.

Other Nebraska Grown.

Silver King

First lear II'is. Seed

Other Nebraska Grown...

Murdock

First Year Wis. Seed...

Other Nebraska Grown.

Cold Resistant Golden Glow .....

Pride of the North

White Cap Yellow Dent

Reid's Yellow Dent..

Improved Leaming...

Iowa Gold Mine

Longfellow Flint

Sanford's White Flint

Red Cob Ensilage (Mio.Grown)....

Evergreen Sweet Fodder (45 lbs.)

\section{N P GN the original}

Soil Inoculator Alfaifa, Sweet Clover, Soys, Clovers, Peas, Beans, Vetch, Cowpeas-all legumes.

15.00

12.50

7.50

(l olit

65.00

29.00

32.00

16.00

14.00

The 1 bu. size will inoculate one bu. or sixty portion.

Wholesale Retail $\begin{gathered}\text { Prices reduced on Soys } \\ \text { and Cow Peas }\end{gathered}$

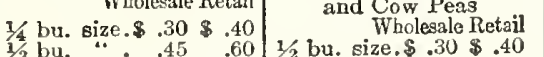

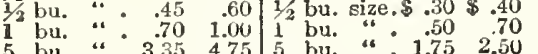
Always state kind of seed-legumes on:y Garden Size (for peas, sweet peas

ald GOLD INOCULATION

For Alfalfa Sw't Clo. and Clo.

1 bu. size, retail price.. 1.00 $1 / 2$ bu. size, retail price.. $\quad .60$ For Soy Beans and Field Peas 5 bu. size, retail price.... 2.00 1 bu. size, retail price.... .50 40 per cent discount to dealer (Name seed wanted for)

\section{OLDS' SEED BARLEY}

Wisconsin Pedigree

Regular Stock ...................... . Sold out

Certified Stock............................ Sold out

Registered Stock ............. Sold out

\section{OTHER SEED GRAIN}

Marquis Spring Wheat

Fancy Montana.....

ITisconsin Grown ................ Sold out

Durum Wheat, Fancy Dakotic

Spring Rye........... Sold out

Flax Seed

Wilt Resistant......................... Sold out

Common_.................................. Sold out

Speltz or Emmer...................... Sold out

Buckwheat

Japanese............................... Sold out

Silver Hull................................ Sold out

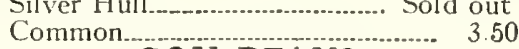

SOY BEANS

(Prices include sacks)

Manchu (Prices include sacks) Sold out

Black Eyebrow ........................... 5.00

Midwest or Hollybrook.......... Sold out

Wisconsin Black .................. Sold out

I to San ................... Sold out

A. K......... 5.00

PEAS AND BEANS
(Prices include sacks)

Field Peas

White Canada, Wis, No, 1 .... Sold out Pedigree Scotch Beauty.... Sold out

Field Beans

White Navy, Wis. C. H. P... Sold out

Red Kidney, IVis. C. H. P........ 8.50

Brown Swedish, Wis. C. H. P..... 7.00

\section{MILLETS}

(Bags extra)

White Wonder............. 4.00

German, Tennessee Cultivated .. 4.25

Common......... 4.25

Siberian $\quad 4.50$

Hog or Broom Corn

OTHER FORAGE CROPS

Sudan Grass.......................... Sold out

Dwarf Essex Rape, Holland Grown 6.75

Early Amber Cane, Northern

Early Amber Cane, Southern

Grown Sold out

White Kaffir Corn............................ 2.75

Milo Maize................................... 2.75

Feterita.................................... 3.00

Mammoth Russian Sunflower... 6.00

Sand Vetch, Fancy Domestic........ 17.00 GRAIN BAGS

Royal River A, per bale 30.00

Onio A, per bale.................................. 29.00 


\section{Poultry Feeds and Supplies, Fertilizers, Tools, Insecticides}

POULTRY AND CHICK FOOD Without Grit)

Per 100 lbs. Per ton
Less than ton Ton or over

Old Gold Poultry Food (100s) $\$ 2.65 \$ 51.00$ Old Gold Chick Food (100s) _... 3.0058 .00

SHELLS AND GRIT (Two Sizes)

Oyster Shells (100s)

Clam Shells (100s)

Pearl Grit (100s)

Lime Grit (100s).

\section{BLATCHFORD FEEDS}

Calf Meal (100s)

Egg Mash (100s)

Chick Mash (100s)

Growing Mash (100s)

3.4566 .50

$4.15 \quad 81.00$

Calf Meal in $50 \mathrm{~s} \$ 2.00$ per ton additional)

(10 lb. sacks Chick Mash $\$ 7.00$ additional)

Chick Mash, Case of 24-4-1b. cartons 6.25

CONKEY BUTTERMILK FEEDS

In sãcks, per 100 pounds 5s $10 \mathrm{~s} \quad 25 \mathrm{~s}$

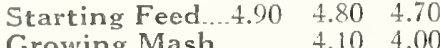
Growing Mash.- $\quad 4.10 \quad 4.00 \quad 3.90$ (Starting Feed in $2 / 2 \mathrm{~s}$ at $\$ 5.05$ per $\mathrm{c}$.

Sacks included Per $100 \mathrm{lbs}$.

Granulated Charcoa!, (Two sizes).. 2.40

Poultry Bone, $(25 \mathrm{~s} \$ 1.00,50 \mathrm{~s} \$ 1.85) 3.50$

Bone Meal, (25s $\$ 1.00,50$ s $\$ 1.85)$... 3.50

Meat Scraps, $(25 \mathrm{~s} \$ 1.25,50 \mathrm{~s} \$ 2.35) .4 .50$

Meat Meal (Tankage)

Alfalfa Meal. No. 1 Medium..Sol on

Steel Cut Oat Meal

Feed Millet

Cracked Corn, Coarse

Sunflower Seed

Feed Wheat, Sacks extra CONKEY'S FLY KNOCKER

Ouarts, per doz

Hall Callon, per doz.

(:allons, per doz

PRA'T'S FLY CHASER

Callons, per doz.

gailon cans, each

\section{CARBOLINEUM}

Gallons, per dozen

5 - gallons, each

o11

OTHER POULTRY SUPPLIES

Utility Poultry Feeders, each

Lawn Park Coops, each

'Babie"' Chick Feeders, each. Automatic Chick Greasers, each. Norwich Air-Lock Fountains, ea

Freezless Fountains, 2 gal., each. 3 gal., each.

Celluloid Leg Bands, per 100

Bandeftes, per 100

Pettey Poultry Punches, per dox. Pratt Renzedies, 25c sizes, per doz. $50 \mathrm{c}$ sizes, per doz.

Conkey $25 \mathrm{c}$. Remedies, per doz. per doz.

Conkey's Y. O. 1 lb. sizes, per doz. 2 lb. sizes, per doz. 10.00 5 lb. sizes, per doz. 21.00

Buttermilk Feeders, per doz.......... 3.75 Automatic Chick Fountains, doz. McCandlish Chick Feeders, 20 in

\section{BEANS}

Sacks included

Beans, Illite Nay, C. IJ P IVis. $100 \mathrm{lbs}$ Darkkedkidney, C.H.P., II is. lightkedkidney, C.H.P., Wis.

Brown Swedish, C.H.P. II is.

FANCY SHELLED POP CORN For Popping)

White Rice

Dwarf Rice .00

5.00

2.90
1.1 .5

Out
8.50

7.50

\section{$\mathrm{N}$}

\section{FERTILIZERS}

Wheat and Corn Grower (2-12-2) Perton

Truck Gardeners' Special (4-8-6) 40.00 Peat Soil Fertilizer (0-12-12) ..... Out

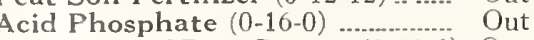
Tobacco and Beet Grower (2-12-6) . Out Bone Meal (11/4-29-0) ................... 42.25

Nitrate of Soda (18-0-0) (In200s) 8500

0 Sheep Manure (In 100 lb. sacks) ....32.00 In 25 lb. sacks) 38.00

38.00

Lawn Lime (In 100 lb. sacks)

Vigoro (4-12-4) (In 100 lb. sacks)....70.00

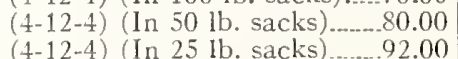
(4-12-4) 5 lb cartons, each

Arcadian Sulyhate of Ammonia

2 lb. boxes, $(35 \mathrm{c})$, per case of 24.- 4.80

5 lb. sacks, $(65 \mathrm{c})$, each

$10 \mathrm{lb}$. sacks, $(1.00)$, each

$50 \mathrm{lb}$. sacks, (3.50), each

\section{OTHER PLANT FOODS}

Oyama, Pl. Food, 25c size, per doz. 2.00 " " " 1.00 size, per doz. 8.00 Watch-Em-Grow, 10c size, per doz. .80 Fern Food,

50 c size, per doz. 4.

Plantpep,

c size, per doz. .80

$25 \mathrm{c}$ size, per doz. 2.00

15c size, per doz. 1.20 50c size, per doz. 4.00

Stim - U - Plant, 15 c size, per doz. 1.20 25 c size, per doz. 2.00 SOILTEX

For Testing Soil for Needed Lime)

Dollar Size, per doz......................... 7.20

GARDEN TOOLS \& SUPPLIES

Planet Jr. Tools, 25 per cent discount.

Hudison Cultivator Hoes, per doz. 8.40

Hudson Garden Plows, each........ 3.00

arker Weeders, 6 inch, each $8 \frac{1}{4}$ inch, each

10 inch, each.

11 inch, each.

12 inch, each

Hero Corn Graders, each

Cyclone Seeders, per doz.

Lawn Settees, 4 foot, each

5 foot, each.

6 foot, each.

Norcross Hoes, 5 Prong; per doz 3 Prong, per doz

Norcross Midget Weeders, per doz. 4.2

Out-U-Kum Weed Pullers, per doz.6.50 Lang Hand Weeders, per doz.

Magic Weeders, Style A, per doz Style B, per doz.

California Pruning Shears, per d

Grasnip Shears, per doz.

Garcen Trowels, Heavy, per doz Light, per doz

Acme Corn Planters

Plain, per doz.

With P. S. A., per doz.

With Fiert. Att., per doz.

.50 Rotary Corn Planters, per doz...

Acme Potato Planters, per doz.

9.00
10.25

10.25
11.00

11.50

14.00

18.40
3.75
.1 .25

4.25

4.50

10.25

1.7 .7

2.50

3.50
7.50

10.00

1.40

40
85

9.75

11.75

2.3 .00
21.50

Invincible Potato Planters, per dz.12.50

Paper Flower Pots, 20 per cent discount

Painted Labels, 20 per cent discount. SPRAYERS

Myers Barrel Spray Pump, each...10.50 Myers Bucket Spray Pump, each.. Armstrong Spray Pumps, each.

Rain King Sprinklers, each

Fountain Lawn Sprinklers, 1 doz.

Half

HudsonPerf. Sprayer, Galv., each Junior Hudson, Galv., each, each Brass, each Continuous Sprayers, per doz. Baby Midget, one pint, per doz. Lenox Sprayers, per doz.

.00 Dickey Dusters, per doz.......

5.00
6.25
B--D Sifters, per doz.

INSECTICIDES

Per lb.

Devoe Paris Green

$1 / 4$ lb. cartons (50 lbs. per case)...\$ Out

$1 / 2$ lb. cartons (50 lbs. per case).... Out

1 lb. cartons (50 lbs. per case)... Out

2 and 5 lb. cartons (50 lbs. per

$$
\text { case) .... }
$$

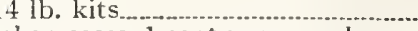

.29

Devoe Dry Arsenate of Lead

$1 / 2 \mathrm{lb}$. cartons (30 lbs. per case)... .26

1 lb. cartons (30 lbs. per case)... .23

4 lb. bags (32 lbs. per case) ........ .16

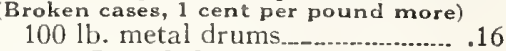

Devoe Dry Calcium Arsenate

$1 / 2$ lb. cartons (30 lbs. per case)... . .21

1 lb. cartons (30 lbs. per case).... .18

4 lb. bags (32 lbs. per case)....... $\quad .12$

(Rroken cases, 1 cent per pound mor

evoe Dry Bordeaux Mixture

$1 \mathrm{lb}$. cartons (30 lbs. per case) ...... .24

4 lb. cartons (32 lbs, per case) -..... $.161 / 2$

$100 \mathrm{lb}$. metal drums.

Devoe Dry Lime SuIfur

1 lb. cans (24 lbs. per case)....... . .21

$5 \mathrm{lb}$. cans (40 lbs, per case) ........ .19

$10 \mathrm{lb}$. cans (40 lbs. per case) ........ $\quad .17$

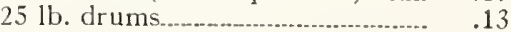

$50 \mathrm{ll}$. drums

$100 \mathrm{lb}$. drums.................... $\quad .11$

(Broken cases, 1 cent per pound more)
(200 lbs. or over, 1 cent per pound less)

200 lbs. or over, 1 cent per pound less)

$1 \mathrm{lb}$. cartons (25 lbs. per case) ..... $\quad .15$

1 lb. cartons (50 lbs. per case)..... $\quad .14$

$5 \mathrm{lb}$. bags (50 lbs. per case) .......... .09

(Brolien cases, 1 cent per pound more)

$\begin{array}{ll}1 \text { lb. pkgs., per doz...................... } & 1.50 \\ 5 \text { lb. pkgs., per doz................. } & 5.50\end{array}$

$121 / 2 \mathrm{lb}$. pkgs., per doz................ 13.50

$100 \mathrm{lb}$. kegs, each...........................

lug Shot

$1 \mathrm{lb}$. cartons, per doz.................... Out

$5,10,25$ lb. bags, per lb.

lack Leaf 40

1 oz. sizes, per doz............ 2.50

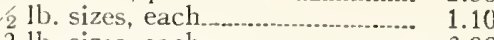

2 1b. sizes, each _........................... 3.00

Black Leaf Nic Dust

5 lb. sizes, each....................... 1.15

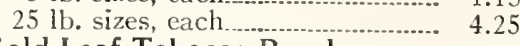

Gold Leaf Tobacco Powder

2 lh. sizes (25c) per doz........... 2.00

$10 \mathrm{lb}$. sizes (85c) each $\quad .60$

$100 \mathrm{lb}$. sizes $(6.50)$ each...................... 5.00

Sulpho Tobacco Soap

oz., per doz.

ormaldehyde, 1 lh. sizes, per doz.

Rat Corn

25 c size, per doz.

1.00 size. per doz.................. 8.00

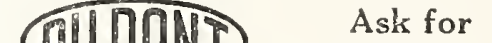

Complete

SEMESAN Marketing

Makes Seeds Healthy Service

Semesan. For Seeds, Bulbs, Roots.

A nationally advertised produrt for

control of seed and soil borne diseases.

Ask for descriptive booklets.

2 oz. size (50c) Der doz.............. 4.00

8 oz. size (1.60) per doz......... 12.80

1 1b. size (2.75) per doz............. 22.00

5 lb. size $(13.00)$ each _ 8.67

25 lb. size (62.50) each........................... 41.67

Semesan, Jr. For Corn.

$4 \mathrm{oz}$. size $(60 \mathrm{c})$ per doz............. 4.80

$1 \mathrm{lb}$ size (1.75) per doz 14.00

$5 \mathrm{lb}$. size $(8.00)$ each $\quad 5.33$

25 lb. size $(37.50)$ each............... 25.00

Semesan Bel. For Potatoes.

$1 \mathrm{lb}$. size (1.75) per doz.

14.00 